\title{
Evaluación de las áreas estratégicas en Pamplona
}

\author{
Henry A Zarta $\mathbf{A}^{1}$; María Esther Rivera ${ }^{2}$; Luis Roberto Sanchez ${ }^{3}$ \\ ${ }^{1}$ Grupo de Investigaciones Agua, Aire y Suelo (GIAAS). Universidad de Pamplona. \\ ${ }^{2}$ Grupo de Investigaciones Ambientales Agua, Aire y Suelo (GIAAS). Universidad de Pamplona. \\ Facultad de Ingenierías y Arquitectura. Programa Ingeniería Ambiental. \\ 3 Grupo de Investigaciones de Recursos Naturales. Universidad de Pamplona Facultad de Ciencias \\ Básicas
}

\section{Resumen}

Con el pasar del tiempo y el rápido avance de la tecnología, el mundo actual está adoptando una tendencia individualista, lo cual solo permite pensar en el "yo", en las comodidades, olvidando aquellos lugares de los cuales se derivan comodidades como las áreas estratégicas ambientalmente importantes. Este proyecto se llevó a cabo entre la Universidad de Pamplona y la Empresa de servicios públicos del Municipio de Pamplona para evaluar las condiciones actuales de la zona de estudio. Se recolectaron muestras en campo con la implementación de seis parcelas distribuidas en dos niveles altitudinales diferentes, subdividiendose en 5 subparcelas; posteriormente se analizó estadísticamente la cobertura vegetal determinando el índice de diversidad y riqueza, mediante el software PAST; se determinó la dominancia entre las 6 parcelas aun estando en diferentes niveles altitudinales, oscilando entre los valores de 0,1 a 0,2 siendo el más alto 0,168 para la parcela P1-3 estos valores similares definen la no existencia de dominancia; Se aprecia una diversidad atenuada, lo cual lleva a analizar el porqué de estos resultados; si retrocedemos 7 y 10 años en el pasado donde se tenía que las actuales áreas estratégicas eran implementadas para realizar actividades agrícolas con siembra de cultivos: papa, fresa, lechuga, papa criolla, en los cuales se aplicaban insumos químicos: fertilizantes, pesticidas, herbicidas, que han predispuesto el terreno facilitando el desarrollo de un grupo de especies más que las otras; otro factor es que el bosque nativo está a una distancia mayor de $2 \mathrm{~km}$ dificultando el transporte de semillas por aves mas no se presenta ausencia de este fenómeno. Se concluye que la zona estudiada ha evolucionado de manera positiva para hoy en día ser un bosque primario estableciendo un clímax de especies.

Palabras clave: Áreas estratégicas, Diversidad, Pamplona, Riqueza, Bosque Primario, Climax.

\section{STRATEGIC ÁREAS EVALUATION IN PAMPLONA}

\section{Abstract}

While going though time and the rapid advancement of the technology, the current world is adopting an individualistic tendency, which allows to think in "me", in the facilities, forgetting those places in which this facilities come from, such as the important strategic enviromental areas like the river Pamplonita steem. Therefore, this project was developed among the University of Pamplona and the Public service of the Municipality of Pamplona to evaluate the 
48

current conditions of the study area. Samples were gathered in field by implementing six plots distributed in two different altitudinales levels, subdivided in 5 subplots; furthermore, the vegetable coverage was analyzed to determine the diversity and wealth index by the implementation of the PAST software; the domination decided between 6 plots even being in altitudinals levels different, ranging between the values from 0,1 to 0,2 being the highest 0,168 for the plot P1-3 these similar values define not domination existence; According to the diversity measuring methods an attenuated diversity is appreciated, which leads to analyzing the reason of these results; if we step back 7 and 10 years in the past where was had that the current strategic areas were implemented to realize agricultural activities with cultivation sowing: potatoes, strawberry, lettuce which chemical inputs were applied: fertilizers, pesticides, herbicides predisposed the area facilitating the development of a group of species more than others; another factor is that the native forest this one at a major $2 \mathrm{~km}$ distance making the seeds transport difficult for birds more does not present absence of this phenomenon to herself. One concludes that the studied area has evolved in a positive way nowadays to be a primary forest establishing a species climax.

Keyword: Strategic área, Climax, Pamplona

*Para citar este artículo: Henry A Zarta A HA;_María Esther Rivera ME; Luis Roberto Sánchez. Evaluación de las áreas estratégicas en Pamplona. Revista Bistua. 2016. 14(1):47-61

+ Autor para el envió de correspondencia y la solicitud de las separatas: Universidad de Pamplona. Facultad de Ingenierías y Arquitectura. Programa Ingeniería Ambiental. Grupo de Investigaciones Ambientales Agua, Aire y Suelo (GIAAS). Ciudadela universitaria, Km 1, Vía Bucaramanga, Pamplona, Norte de Santander, Colombia. Correo electrónico: maes@unipamplona.edu.co 


\section{Introducción}

Hoy en día con el desarrollo tecnológico, el mundo actual adopta una tendencia individualista, lo cual solo permite pensar en el "yo", en las comodidades, pero se olvidan de aquellos lugares, donde se derivan todas las comodidades; por lo anterior surgió la necesidad de realizar presente investigación en el municipio de Pamplona Norte de Santander Colombia, siendo pioneros en el estudio de áreas estratégicas en la zona innovando con la información recopilada y metodologías aplicadas para conseguir un análisis más preciso, y decisivo en la toma de decisiones encaminadas al mejoramiento de la cobertura vegetal y la protección de las especies de la zona; se implementaron metodologías como muestreos de plantas leñosas y para el análisis de la información y procesamiento de datos se aplicaron diferentes autores como Índice de Shannon, Índice de Simpson, Índice de Margaleff, Índice de BergerParker. Para la practicidad y mejoramiento de la eficiencia del estudio se implementó el uso del software PAST para el análisis estadístico y la implementación de tablas dinámicas en el programa Excel.

Por diversidad de especies se entiende la variedad de especies existentes en una región. Esa diversidad puede medirse de muchas maneras, y los científicos no se han puesto de acuerdo sobre cuál es el mejor método. El número de especies de una región--su "riqueza" en especies es una medida que a menudo se utiliza, pero una medida más precisa, la "diversidad taxonómica" tiene en cuenta la estrecha relación existente entre especies ${ }^{1}$.

\section{Índices de diversidad}

Autores como 2, 3, 4 coinciden en señalar que el índice de diversidad está formado por dos componentes: el número de especies o riqueza de especie y la abundancia o equilibrio de especie.

Aplicación de los índices un problema de muestreo

Muchas de las investigaciones en ecología tienden a caracterizar una comunidad y en la mayoría de las ocasiones se desea obtener información de áreas o superficies muy grandes, dada la usual imposibilidad de hacer un censo se utilizan muestras. Si bien es cierto se ha llegado a dar una interpretación teórica a los índices como medidas de diversidad se necesitan estudios empíricos del desempeño de sus estimadores muestrales ${ }^{5}$

Analizando con detenimiento el problema de la estimación de los índices, encontraremos situaciones no muy cómodas para aplicar la teoría de muestreo, en principio no se cuenta con un marco muestral o su construcción sería muy costosa y el vector de 
50

abundancia de especie $\pi$ es igualmente desconocido ${ }^{6}$

\section{Índices de diversidad de especies}

El índice de Shannon se basa en la teoría de la información y por tanto en la probabilidad de encontrar un determinado individuo en un ecosistema. El índice contempla la cantidad de especies presentes en el área de estudio (riqueza de especies), y la cantidad relativa de individuos de cada una de esas especies (abundancia) $^{7}$

Es una de las medidas de diversidad relacionadas con la teoría de información. Estas medidas parten del supuesto de que una comunidad (ensamblaje de organismos presentes en un hábitat) es análoga a un sistema en la cual existe un número finito de individuos, los cuales pueden ocupar un número, también finito- de categorías (especies, análogo de estados) ${ }^{7}$

La estadística para describir esta situación: un sistema con un número finito de individuos y de categorías (especies); sin restricciones en cuanto al número de especies ni de individuos por categoría (especie), está dada por la Fórmula; equivale a la incertidumbre acerca de la identidad de un elemento tomado al azar de una colección de $\mathrm{N}$ elementos distribuidos en $\mathrm{s}$ categorías, sin importar el número de elementos por categoría ni el número de categorías. Dicha incertidumbre aumenta con el número de categorías (riqueza) y disminuye cuando la mayoría de los elementos pertenecen a una categoría ${ }^{1}$
Este índice fue desarrollado para medir la cantidad de información que se puede transmitir, donde pi representa la proporción (o abundancia relativa) de cada especie en la población y "log" es la abreviatura del logaritmo (la base del logaritmo no importa, puede ser base 10 (decimal), base 2 (binaria) o base $" \mathrm{e} "=2.7182 \ldots$, la base de los logaritmos naturales, es la más utilizada actualmente). La sumatoria es sobre las "S" especies $(i=1,2, \ldots, S)$ de la población. Si llamamos ni al número de individuos de la especie "i" y $\mathrm{N}$ a la población total de la colección, entonces $\mathrm{pi}=\mathrm{ni} / \mathrm{N}$. El tamaño de la población (N) se calcula sumando los individuos de todas las especies, es decir N. ${ }^{8}$

La Fórmula de Shannon-Weaver que es la forma en la cual normalmente se presenta la diversidad de especies basada en la teoría de información; De esta forma, el índice contempla la cantidad de especies presentes en el área de estudio (riqueza de especies), y la cantidad relativa de individuos de cada una de esas especies (abundancia) ${ }^{9}$. Este índice se representa normalmente como $\mathrm{H}^{\prime \prime}$ y se expresa con un número positivo, que en la mayoría de los ecosistemas naturales varía entre 1 y 5 . Excepcionalmente puede haber ecosistemas con valores mayores como los bosques tropicales, arrecifes de coral), o menores (algunas zonas desérticas). La mayor limitante de este índice es que no tiene en cuenta la distribución de las especies en el espacio $^{1}$ 
51

\section{Índice de Simpson}

Los índices de dominancia se basan en parámetros inversos a los conceptos de equidad puesto que toman en cuenta la dominancia de las

especies con mayor representatividad, para lo cual el índice más común para utilizar es el índice de Simpson. El índice de dominancia de Simpson (también conocido como el índice de la diversidad de las especies o índice de dominancia) es uno de los parámetros que nos permiten medir la riqueza de organismos. En ecología, es también usado para cuantificar la biodiversidad de un hábitat. Toma un determinado número de especies presentes en el hábitat y su abundancia relativa. ${ }^{6}$

A medida que el índice se incrementa, la diversidad decrece. Por ello el Índice de Simpson se presenta habitualmente como una medida de la dominancia, como se acaba de indicar. Por tanto, el índice de Simpson sobrevalora las especies más abundantes en detrimento de la riqueza total de especies. Entonces entre más aumente el valor a uno, la diversidad disminuye. ${ }^{6}$

Este índice de Simpson de dominancia $D=p^{\wedge} 2$ estima si en un área determinado hay especies muy dominantes al sumar términos al cuadrado le da importancia a las especies muy abundantes y por tanto la dominancia dará una cifra alta, cercana a uno que es el valor máximo que toma el índice, si la dominancia es alta la diversidad será baja como ya fue mencionado ${ }^{7}$.

El índice de Simpson precisa el valor de pi. Siendo $\mathrm{pi}=\mathrm{ni} / \mathrm{N}$, donde $\mathrm{ni}$ es el Bistua Revista de la Facultad de Ciencias Basicas .Universid número de individuos de la especie "i" y $\mathrm{N}$ es la abundancia total de las especies. Con otras palabras, pi es la abundancia proporcional de la especie „": Si bien este índice depende de la cantidad de categorías que es posible reconocer, da También una idea de homogeneidad general partiendo de la base de que un sistema es más diverso cuanto menos dominancia de especies hay, y la distribución es más equitativa. Tomando en cuenta que el valor mínimo para este índice es 1 que indica que no hay diversidad y que la dominancia es alta. ${ }^{10}$

Índice de Margaleff

La riqueza de especies proporciona una medida de la diversidad extremadamente útil. En general, no solamente una lista de especies es suficiente para caracterizar la diversidad, haciéndose necesaria la distinción entre riqueza numérica de especies, la que se define como el número de especies por número de individuos especificados o biomasa y densidad de especies, que es el número de especies por área de muestreo. Para esto se pueden utilizar ciertos índices, usando algunas combinaciones como el número de especies y el número total de individuos sumando todos los de las especies ${ }^{11}$

La medición de la riqueza específica es la forma más sencilla de medir la biodiversidad ya que solo se basa en el número de especies presentes sin tomar en cuenta el valor de importancia el índice que se utiliza para medir la riqueza especifica fue el índice de Margaleff, el cual transforma 
52

el número de especies por muestra a una proporción en la cual las especies son añadidas por expansión de la muestra $^{12}$. Además, es una medida utilizada en ecología para estimar la biodiversidad de una Comunidad con base a la distribución numérica de los individuos de las diferentes especies en función del número de individuos existentes en la muestra analizada, esenciales para medir el número de especies en una unidad de muestra ${ }^{11}$. Donde Valores inferiores a 2,0 son considerados como relacionados con zonas de baja diversidad (en general resultado de efectos antropogénicos) y valores superiores a 5,0 son considerados como indicativos de alta biodiversidad ${ }^{11}$

\section{Índice de Berger-Parker (B)}

El índice de Berger Parker Mide la dominancia de la especie o taxón más abundante. Este índice adquiere valores comprendidos entre 0 y 1 (0\% y 100 $\%) .{ }^{13}$ Además, es indicador de los mismos impactos que el índice de Simpson: polución orgánica, degradación en la morfología y degradación general. Una forma de medir la dominancia en forma simple es mediante el Índice de Berger- Parker, que tiene la virtud de la simplicidad de su cálculo, expresando la importancia proporcional de las especies más abundantes $^{12}$

\section{Equidad}

La equitatividad (E) indica que tan uniformemente están distribuidos los individuos entre las especies ${ }^{14}$; Se puede medir comparando la diversidad observada en una comunidad contra la diversidad máxima posible de una comunidad hipotética con el mismo número de especies. Puede demostrarse que cuando $\mathrm{pi}=1 / \mathrm{S}$ para toda pi, se alcanza la uniformidad máxima siendo pi la proporción del número de individuos de la especie i con respecto al total $(\mathrm{ni} / \mathrm{Nt})$ ) para lo cual generalmente el índice de equidad más apropiado es el índice de Shannon ${ }^{15}$

\section{Formas de analizar la diversidad}

En ecología se pueden hacer estudios de diferentes formas. Los estudios pueden ser de tipo descriptivo, comparativo, observacional y experimental. Los estudios descriptivos son generalmente exploratorios y no tienen una hipótesis a prioritaria. El objetivo de estos estudios es obtener información acerca de un fenómeno o sistema del cual previamente se tenía ninguna o muy poca información ${ }^{16}$.

Los estudios comparativos se deben realizar en sistemas de los que se tiene cierta información y cuando se tiene una 0 varias hipótesis de antemano. El objetivo de este tipo de estudio es obtener la información necesaria para someter a prueba las hipótesis. Los estudios observacionales se basan en información obtenida del sistema en su estado original; generalmente no se hace ninguna manipulación del sistema y los estudios experimentales consisten en manipular o modificar, de manera particular, un determinado sistema 0 ambiente (tratamiento experimental). La información que interesa es, precisamente, la respuesta del sistema al tratamiento ${ }^{16}$ 
53

Los índices han sido y siguen siendo muy útiles para medir la vegetación. Si bien muchos investigadores opinan que los índices comprimen demasiado la información, además de tener poco significado, en muchos casos son el único medio para analizar los datos de vegetación. Los índices de biodiversidad son los más utilizados en el análisis comparativo y descriptivo de la vegetación ${ }^{16}$.

\section{Diversidad floristica}

En Ecología el término diversidad florística ha designado tradicionalmente un parámetro de los ecosistemas (aunque se considera una propiedad emergente de la comunidad) que describe su variedad interna. El concepto resulta de una aplicación específica de la noción física de información, y se mide mediante índices relacionados con los habitualmente empleados para medir la complejidad. El uso tradicional se encuentra ahora inmerso en una batalla por conservar su significado frente al, mucho más político que científico, concepto de biodiversidad. La diversidad de un ecosistema depende de dos factores, el número de especies presente y el equilibrio demográfico entre ellas. Entre dos ecosistemas hipotéticos formados por especies demográficamente idénticas (el mismo número de individuos de cada una, algo que nunca aparece en la realidad) consideraríamos más diverso al que presentara un número de especies mayor. Por otra parte, entre dos ecosistemas que tienen el mismo número de especies, consideraremos más diverso al que presenta menos diferencias en el número de individuos de unas y otras especies. Desde hace ya bastante tiempo la mayoría de los ecólogos han coincidido en que la diversidad de especies debe ser distinguida en al menos tres niveles: La diversidad local

ó diversidad $\alpha$, la diferenciación de la diversidad entre áreas o diversidad $\beta$ y la diversidad regional ${ }^{7,13}$

\section{Muestreos de plantas leñosas}

Esta metodología se utiliza para determinar la riqueza de especies de plantas leñosas y suministra información de la estructura de la vegetación. Fue propuesta por ${ }^{17}$ y ha sido ampliamente utilizada en el Neotrópico, permitiendo realizar buenas comparaciones. Entre sus desventajas se encuentra que no suministra información completa de la composición de especies, por lo que no se pueden efectuar comparaciones de similitud, y requiere de la colección e identificación de muchas especies. Sin embargo, suministra información importante y complementaria a la de muestreos de Rubiaceae y Melastomataceae.

Este método consiste en censar, en un área de $0.1 \mathrm{ha}$, todas los individuos cuyo tallo tenga un diámetro a la altura del pecho (DAP medido a $1.3 \mathrm{~m}$ desde la superficie del suelo) mayor o igual a $2.5 \mathrm{~cm} .{ }^{17}$

\section{Metodologia}

Para la determinación de cantidades exactas de las áreas de importancia estratégica del municipio de Pamplona se realizó el trabajo de campo empleando diferentes equipos para la toma de puntos esenciales al momento 
54

de la localización exacta de las áreas. Así como planos y documentos suministrados por la empresa de servicios públicos del municipio de Pamplona (EMPOPAMPLONA). Se realizaron 10 salidas de campo para la toma de los diferentes puntos de referencia, con los cuales fue posible plasmar en la cartografía una aproximación muy real de los linderos de la zona; esta elaboración cartográfica fue posible con el uso del GPS Garmin montana 650 y la implementación del programa ArcGIS v.10.

En la evaluación de las condiciones actuales de las áreas de importancia estratégica; se llevó a cabo in-situ para lograr mayor precisión y conseguir plasmar la realidad que se vive en la zona de estudio. De igual manera se llevó a cabo un análisis estadístico para la cobertura vegetal de la zona mediante el software PAST aplicados a los datos recopilados en las anteriores actividades e investigaciones bibliográficas que se realizaron. Para la toma de muestras florísticas se realizaron parcelas de $12,5 \mathrm{~m} \times 4 \mathrm{~m}$ y $25 \mathrm{~m} \times 4 \mathrm{~m}$. Ademas, se censaron individuos con DAP mayor o igual a 1 $\mathrm{cm}$, pues con esta modificación se obtiene unamejor representación de los estratos inferiores (sotobosque). Una vez finalizada la fase de campo, se debe llevar a cabo una lista de las especies o morfoespecies registradas en los muestreos con base en las colecciones realizadas.

A partir de la tabla base en Excel y utilizando algunos comandos como el de Tabla "Asistente para tablas dinámicas" o "filtro", se organizan los datos para obtener información de la riqueza total en0.1 ha (número de especies/0. 1 ha), densidad total (número total de individuos/0.1 ha), área basal total (sumatoria de las áreas basales de todos los individuos/0.1 ha) y las listas de las familias, géneros y especies.

Igualmente, con los datos organizados se deben calcular los diferentes parámetros estructurales para cada una de las especies registradas en el muestreo. Estos parámetros son: frecuencia, frecuencia relativa, abundancia, abundancia relativa, cobertura y cobertura relativa. Con estos parámetros se calcula el

Índice de Valor de Importancia (IVI) de cada una de las especies en el muestreo. EI IVI es un estimativo de cuán dominante es cada especie con respecto a la totalidad de las especies registradas en el muestreo.

Otro tipo de información estructural que se debe obtener con los datos es la distribución de individuos por clases de alturas y de tallos por clases diamétricas. Para esto se deben establecer los rangos de diámetros o de altura y determinar cuántos individuos o tallos se encuentran en cada uno de estos rangos, luego se realizan gráficas de barras. ${ }^{19}$

Para la obtención del índice de diversidad de especies se precisa conocer tanto el número de especies de una familia (ni) como el número total de especies dentro de la parcela, para lo cual se trabajó con tablas dinámicas de Excel, dividiendo la base Bistua Revista de la Facultad de Ciencias Basicas .Universidad de Pamplona. ISSN 0120-4211 
55

de datos para las 6 parcelas con sus especies e individuos. Para obtener el número total de especies $(\mathrm{N})$ se realizó la sumatoria de todas las especies de las familias pertenecientes a una misma parcela. Procediendo de la misma manera para las 6 parcelas. De igual manera, en el cálculo de los índices de biodiversidad se implementó el software PAST con el cual se calculó el dendrograma a partir del análisis de clúster y todos los índices

\section{Resultados y Discusión}

De la tabla 1 se observa que la dominancia entre las 6 parcelas aun estando en diferentes niveles altitudinales oscila entre los valores de 0,1 a 0,2 siendo el más alto 0,168 presente para la parcela $\mathrm{P} 1-3$ estos valores tan similares permiten definir que la no existe de ninguna especie que domine sobre la otra, lo cual se puede corroborar con los datos obtenidos en la fila de equitabilidad, en el rango para la obtención de los valores es de 0 a 1 y observando los resultados obtenidos los valores oscilan entre 0,69 a 0,9 , mostrando similaridad entre ellos, en algunas parcelas como P2-2 y P2-3 se observan valores de 0,9 lo que impulsaría a pensar que tienden a dominar, lo cual no sería correcto debido a que no son espacios muy separados entre los valores de las otras parcelas.

Para corroborar lo dicho anteriormente se presentan los resultados de Fisher alpha el cuyos valores oscilan entre $1 \mathrm{a}$ 10 y en los datos que se observan entre las diferentes parcelas se aprecia la similaridad entre ellos excepto la parcela P2-1 en la cual observamos un valor de 4,35 el cual se obtiene de esta manera a razón de la menor cantidad de especies presentes en ella la cual es de 15 en 132 individuos muestreados lo cual nos generó este resultado.

El índice de dominancia de Simpson es uno de los parámetros que permite medir la riqueza de organismos. En ecología, también usado para cuantificar la biodiversidad de un hábitat. Toma un determinado número de especies presentes en el hábitat $y$ su abundancia relativa ${ }^{6}$

En el grafico 1. se reitera la similaridad entre los valores obtenidos en Simpson, los cuales nos conlleva a decir que en los sitios muestreados no existe una especie que domine sobre las otras, esto puede ser causa de que el sistema en estudio ha alcanzado o está muy cerca de un equilibrio de especies está en su clímax, la competencia para lograr sobrevivir y eliminar a los contrincantes ha disminuido considerablemente, encontramos una estabilidad en los microclimas, entre las especies de lo cual podemos decir que se ha vuelto un bosque primario.

Gráfico 1. Índice de dominancia según Simpson

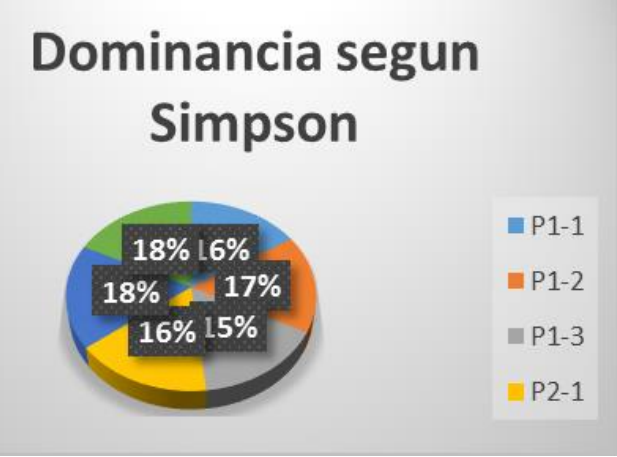

Fuente: Autores 2014. 
56

Para analizar la diversidad de especies se tuvo en cuenta los datos resultantes de los métodos de Margalef y Shannon H. Según los valores obtenidos en las parcelas muestreadas (ver gráfico 2) oscilan entre 2,8 a 4,9 se deduce una diversidad media de especies si nos enfocamos en especial en la parcela P1-3 observamos una diversidad menor que en las demás; aunque la diversidad no es baja, no se puede asegurar que existe alta diversidad, el termino correcto sería una diversidad media considerable.

Grafico 2. Indice de diversidad según Margalef

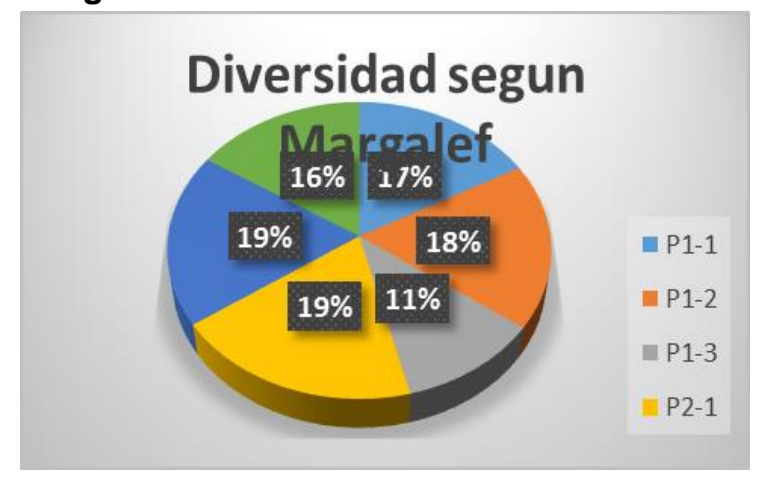

Fuente: Autores, 2014.

En la gráfica 3 se corroboran los resultados observados, en la metodología de Shannon $\mathrm{H}$ en la cual los valores oscilan de 1 a 5 y en nuestros sitios muestreados nos encontramos con valores medios registrándose entre 2,02 a 3,1 lo cual nos reitera una diversidad media considerable.

Gráfico 3. Índice de diversidad según Shannon

\section{Indice de diversidad} segun shannon

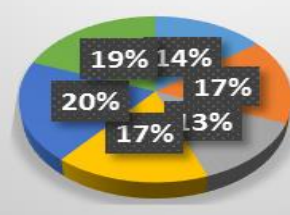

$=P 1-1$
$=P 1-2$
$=P 1-3$
$=P 2-1$
$=P 2-2$

Fuente: Autores, 2014.

Se observó una diversidad atenuada, lo cual permitió analizar el porqué de estos resultados; si retrocedemos 7 y 10 años en el pasado apreciamos que las actuales zonas de protección como lo son las áreas estratégicas eran implementadas para realizar actividades agrícolas entre las cuales podíamos encontrar la siembra de cultivos de papa, fresa, lechuga, papa criolla, entre otros. En estos tipos de cultivos se utilizan insumos químicos como los fertilizantes, los pesticidas, herbicidas, venenos, entre otros, los cuales con el paso del tiempo y a medida que estas áreas fueron adquiridas por el municipio predispusieron el terreno a facilitar el desarrollo de un grupo de especies más que las otras; otro factor de influencia es que el bosque nativo de la zona se presenta a una distancia mayor de $1 \mathrm{~km}$ lo que dificulta el transporte de semillas por las aves mas no se presenta ausencia de este fenómeno.

Con respecto a los resultados obtenidos del análisis de clúster, se observa la notable diferencia entre grupos de parcelas muestreadas formandose de la sigueine manera:

- Grupo 1: parcela 1-1, parcela 12, parcela 1-3 
- Grupo 2: parcela 2-1, parcela 22, parcela 2-3

Imagen 1. Dendrograma o Cluster de especies de la zona de estudio
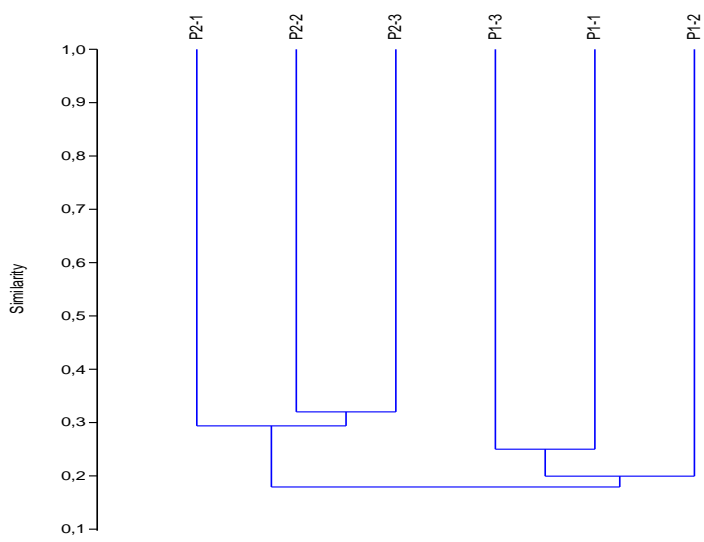

Fuente: Autores, 2014.

De la imagen 1 se observa que la realidad de la zona estudiada, presento dos ecosistemas altamente marcado, pajonal-frailejonal y pajonal-transicional; el dendrograma muestra una similitud entre los 2 grupos formados de un 18\% (dieciocho por ciento) una similaridad muy baja, pero entre los grupos mayores se conformaron subgrupos de la siguiente manera:

Grupo1: grupo 1.1 formado por las parcelas $P$ 1-2 y $P$ 1-3, grupo 1.2 conformado por la parcela $P$ 1-1 y el grupo 1.1

Grupo 2: grupo 2.1 conformado por las parcelas P 2-1 y $\mathrm{P}$ 2-2, grupo 2.2 conformado por la parcela P 2-3 y el grupo 2.1

Los subgrupos conformados se deben a la presencia de manera mas notoria en cantidad de ciertas especies, por tal motivo encontramos similitudes de $36 \%$ grupo 2.1 y $33 \%$ grupo 1.1 .

Por otra parte, en la imagen se aprecia que el grupo 1 presenta mayor similitud entre sus componentes que el grupo 2 lo que lo vuelve un ecosistemas aun mas estable y definido.

Con respecto al Análisis del índice de valor de importancia ambiental (IVI) es necesario tener en cuenta que cuando pensamos en cobertura vegetal el primer pensamiento que nos surge es "gran cantidad de plantas", pero... ¿basta con saber que hay muchas plantas?, la respuesta es no. En cualquier zona siempre encontraremos especies de plantas que tienden a marcar mayor incidencia en la zona, estas son aquellas que los próximos resultados analizados presentan.

Del grafico 4 se infiere que para un análisis global de la zona estudiada, las especies con mayor importancia ecológica según el IVI (ver tabla 2) son: Hypericum-sp con 52,66\%, Arcytophyllum nitidum con $35,29 \%$, Baccharis rupicola con $26,06 \%$, Gaultheria anastomosans con $19,84 \%$, Hypericum cardonae con $17,00 \%$, Espeletia-sp con $15,14 \%$ y las de menor importancia fueron: Berberis-sp con $3,15 \%$ y Hesperomeles obtusifolia con $2,91 \%$.

Grafico 6. Valor de importancia ambiental parte media-baja

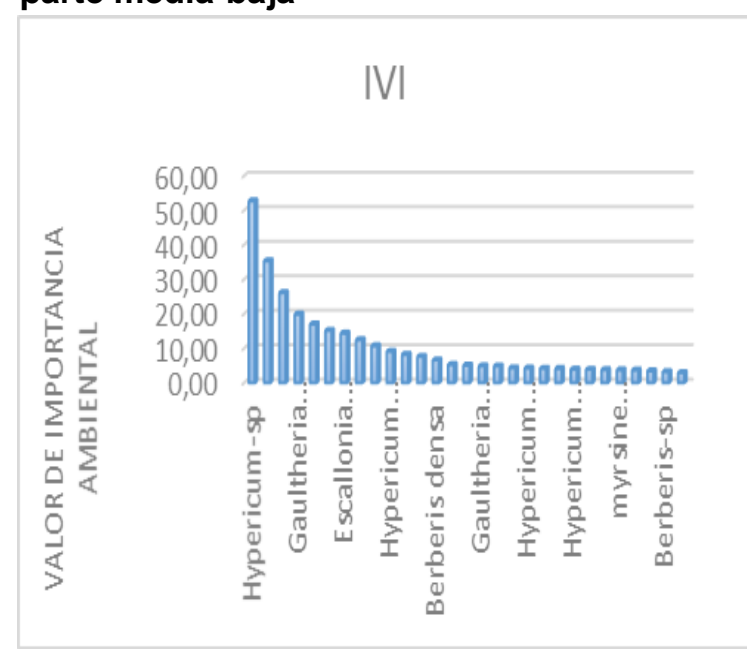


58

Además, se observa que en la parte alta de la zona estudiada se aprecia un valor de importancia alto para las especies: Arcytophyllum nitidum con $64,59 \%$, Baccharis rupicola con 45,34\%, Hypericum-sp con 40,70\%, Hypericum cardonae con $32,73 \%$, Espeletia-sp con $29,21 \%$, y las de menor importancia fueron: Vaccinium-sp con 9,42\% y Hypericum suniperinum con $8,45 \%$. (ver grafico 5)

Grafico 5. Valor de importancia ambiental parte alta

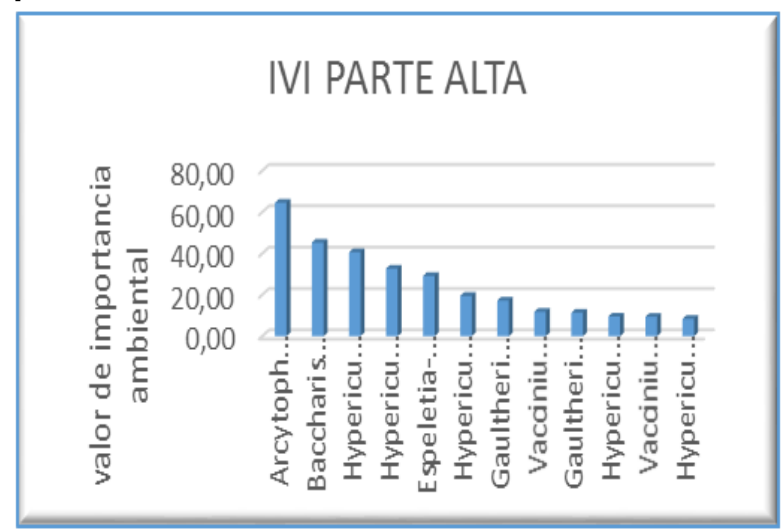

Fuente: Autores, 2014.

En el grafico 6 se aprecia que en la parte media-baja de la zona estudiada encontramos valor de importancia ambiental alto para las siguientes especies: Hypericum-sp con 68,26\%, Gaultheria anastomosans con 27,48\%, Escallonia myrtilloides con 26,56\% y Diplostephium rosmarinifolius con
$22,83 \%$ y las de menor importancia fueron: Berberis-sp con 5,28\% y Hesperomeles obtusifolia con 4,80\%. Grafico 6. Valor de importancia ambiental parte media-baja

IVI PARTE MEDIA-BAJA

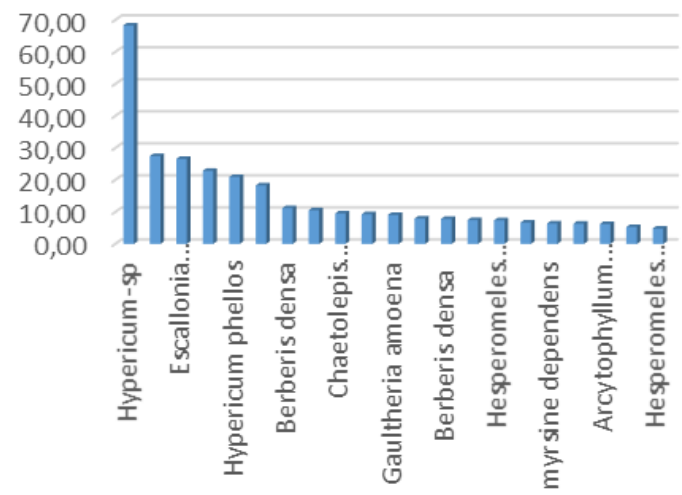

Fuente: Autores, 2014.

En el grafico 6 sea precia una disminución en las especies procesadas, esto se debe a que se aplicaron filtros en las tablas para procesar solo las especies leñosas; la especie Espeletia-sp es considerada como semileñosa pero debido a que uno de los ecosistemas estudiados es pajonal-frailejonal se incluyeron en el estudio. (Ver Taba 3)

Según las especies encontradas en la zona fue necesario enfocarse en la Espeletia-sp, teniendo en cuenta que es una especie amenazada en Colombia, la cual debe ser vigilada de manera sigilosa debido a que las personas sin escrúpulos solo están interesadas en el bienestar personal sin importarles los prejuicios que puedan generar a la comunidad (Ver Tabla 4).

Aunque en las zonas muestreadas no se detectaron Cederla odorata y Quercus humboldtii en algunas partes muy específicas se encontró presencia 
59

de ellas lo cual nos debe guiar a la toma de medidas preventivas en la zona.

\section{Conclusión}

Las áreas estratégicas del municipio de Pamplona ya adquiridas presentan una gran mejoría frente a las posibles áreas por adquirir en lo relacionado con el aumento de la cobertura vegetal; según los análisis de Margalef y Shannon, los cuales arrojaron resultados entre los rangos de (2-5), (2-3) respectivamente, mostrando valores de diversidad media que en años anteriores no se apreciaban; además se encontró ecosistemas muy equilibrados, los cuales presentan características propias de estar en un clímax de especies, corroborándolo con los resultados de Simpson que se aprecian en un rango de (0.8-0.9), con base a los resultados obtenidos en el estudio, se determinó que la cobertura vegetal de la zona presenta claramente la mayor cantidad de características propias de un bosque primario

\section{Agradecimientos}

A la Empresa de Servicios Públicos de la Ciudad de Pamplona EMPOPAMPLONA, por el suministro de información y personal necesario durante la investigación.

\section{Referencias bibliográficas}

1.Moreno, C. "Métodos para medir la biodiversidad". M \& T-Manuales y tesis SEA, Vol. 1. Zaragoza, 84 pp. (Internet) 2001. 20/11/2014. Disponible en: http://www.google.com.bo/search?hl=es\&q= metodos+para+medir+biodiversidad

2. Krebs, C.J.. Ecological Methodology, 2nd ed. Addison-Wesley Educational Publishers, Inc. 1999.
3. Bouza, Carlos N. / Daniel Covarrubias. Estimación del Índice de Diversidad de Simpson en $\mathrm{m}$ Sitios de Muestreo. Revista Investigación Operacional: Vol. 26, No. 2; 2005.

4. Ludwig A. / Reynolds F. Statical Ecology: A Primer on Methods and Computing (internet). A WileyIntercience Publication. (02/2011; 25/10/2014). 1988. Disponible en: http://www2.ib.unicamp.br/profs/thoma s/NE002 2011/maio12/LR\%201988\%2 0StatEcol\%20sel1.pdf

5. Magurran, A. Ecological diversity and is measurement. $41-42$ pp. 2001.

6. Pielou E.C. An Introduction to mathematical Ecology (Internet). Wiley Interscience. New York, EE.UU. 98 pp. (04/04/2009; 18/11/2014). Disponible en: http://rev-inv-

ope.univparis1.fr/files/26205/IO-26205-

9.pdf

7. Smith L.R. 2001. Ecología (Internet). Madrid. Pearson Educación. 62 - 64 pp. (11/06/2009; 25/10/2014). Disponible en: http://redalyc.uaemex.mx/redalyc/pdf/449/

44954228.pdf

8. Halffter, Ezcurra. La Diversidad Biológica de lberoamérica I (Internet).

Primera edición. D.R. (c) Instituto de Ecología, A.C.1992. (2005; 28/10/2014). Disponible en: http://uniciencia.ambientalex.info/infoCT/Di vbioibemx.pdf

9. Pérez Irma Rosas / Carranza Ortiz Gabriela / Nava Cruz Yolanda / Larqué Saavedra Alfonso. La percepción sobre la conservación de la cobertura vegetal. P. 123-140.

10. Lampretch. Ensayo sobre métodos para el análisis estructural de los bosques tropicales. Acta científica venezolana. 1962.

11. Margalef, R. 1969. Diversity and stability: a practical proposal and a model pof inter. deansendence (Internet). Brookhaven 
60

Symp. Biology. 22: 25-37.1969. Disponible en:

http://www.aslo.org/bulletin/margalef pubs. pdf

12. Miller, Kenton. En busca de un nuevo equilibrio: lineamientos para incrementar las oportunidades de conservar la biodiversidad a través del manejo biorregional. Washington D.C. World Resources Institute; 1996.

13. Orellana Lara Jhosmar. Determinación del índice de diversidad florística arbórea en las parcelas permanentes de muestreo del Valle de Sacta (Internet). Universidad Mayor de San Simón, Facultad de Ciencias Agrícolas Forestales y Veterinaria, Escuela de Ciencias Forestales. Cochabamba Bolivia. 2009.12/11/2014.Disponible en: http://www.posgradoesfor.umss.edu.bo/bole tin/umss/05PASANTIAS/6pasantia.pdf 14. Clements, W.H. and M.C. Newman. Community Ecotoxicology. John Wiley and Sons, Chichester, United Kingdom, 336 pp 2002.

15. G CANCINO, D BARBOSA, C DíAZ (2012). Diversidad genética de especies silvestres y cultivadas de Rubus I. de los municipios de Pamplona y Chitagá, región Nororiental de Colombia. Revista Bistua, Vol. 10, no.1, 80-89.

16. Natenzon Claudia. Áreas protegidas, asentamientos locales y desarrollo: el Caso del Parque Nacional Baritú, Buenos Aires. Instituto de Geografía, Facultad de Filosofía y Letras, Universidad de Buenos Aires; 1993.

17. GS Otero (2013). Utilización vinícola de la Opuntia dillenii. Revista Bistua, Vol. 11, no.2.

18. Mostacedo B. Manual de Métodos Básicos de Muestreo y Análisis en Ecología Vegetal. Santa Cruz, Bolivia pag.2 - 8; 2000.

19. Villarreal H. / M.Alvarez / S. Cordoba
Mendoza / M. Ospina / A.M Umaña. Manual de Métodos para el Desarrollo De Inventarios De Biodiversidad. Instituto de Investigación de Recursos Biológicos Alexander von Humboldt. Bogotá, Colombia. 236 p. 2004. 20. Moreno, L., Muñoz Prieto, E., \& Casanova, H. 2015. Flocculation with Chitosan of Microalgae Native of the Colombian Plateau. CIENCIA EN DESARROLLO, 6(1): 25-32.

21. Cárdenas L., D / N.R. Salinas (eds.). Libro Rojo de plantas de Colombia. Especies maderables amenazadas: primera parte. Volumen 4. Instituto Amazónico de Investigaciones Científicas SINCHI-Ministerio de Ambiente, Vivienda y Desarrollo Territorial. 232 PP.; 2007

22. Daniel Covarrubias, Díaz Piedrahita, S. / P. Pedraza / N. García / E. Calderón / G. Galeano. Frailejones (Espeletiinae), Libro Rojo de Plantas Fanerógamas de Colombia. Volumen 2. Calderón, E., G. Galeano \& N. García (eds.): 2004. 


\begin{tabular}{|l|l|l|}
\hline Chaquiro & $\begin{array}{l}\text { Retrophyllus } \\
\text { rospigliosii }\end{array}$ & Podocarpaceae \\
\hline
\end{tabular}

Tabla 1. Cálculo del índice de valor de importancia (IVI)

Fuente: Autores, 2014

61

\begin{tabular}{|l|l|l|l|l|l|l|}
\hline $\begin{array}{l}\text { Índice } \\
\text { diversidad }\end{array}$ & $\mathrm{P} 1-1$ & $\mathrm{P} 1-2$ & $\mathrm{P} 1-3$ & $\mathrm{P} 2-1$ & $\mathrm{P} 2-2$ & $\mathrm{P} 2-3$ \\
\hline Taxa-S & 25 & 24 & 15 & 26 & 27 & 23 \\
\hline individuals & 244 & 140 & 132 & 154 & 210 & 223 \\
\hline Dominance_D & 0,1509 & 0,1129 & 0,1684 & 0,1304 & 0,1 & 0,1 \\
\hline Simpson_1_D & 0,8491 & 0,8871 & 0,8316 & 0,8696 & 0,9 & 0,9 \\
\hline Shannon_H & 2,23 & 2,56 & 2,027 & 2,576 & 3,1 & 2,9 \\
\hline Margalef & 4,366 & 4,654 & 2,867 & 4,963 & 4,9 & 4,1 \\
\hline Equitatibility_J & 0,6928 & 0,8057 & 0,7485 & 0,7907 & 0,9 & 0,9 \\
\hline Fisher_alpha & 6,978 & 8,337 & 4,356 & 8,965 & 8,2 & 6,4 \\
\hline
\end{tabular}

Tabla 2. Índices de Diversidad de la zona de estudio

Fuente: Autores, 2014

\begin{tabular}{|c|c|c|c|c|c|c|}
\hline \multirow{2}{*}{ Genero } & \multicolumn{5}{|c|}{ Numero especies amenazadas } & \multirow{2}{*}{$\begin{array}{l}\text { Total especies } \\
\text { en Colombia }\end{array}$} \\
\hline & $\mathrm{CR}$ & RN & VU & Total & $\%$ & \\
\hline Espeletia & 6 & 11 & 9 & 26 & 58 & 45 \\
\hline espeletiopsis & 1 & 3 & 2 & 6 & 40 & 15 \\
\hline Libanothamnus & 1 & 2 & 0 & 3 & 60 & 5 \\
\hline Paramiflos & 0 & 0 & 1 & 1 & 100 & 1 \\
\hline Ruilopezia & 0 & 0 & 0 & 0 & 0 & 1 \\
\hline Tamania & 0 & 1 & 0 & 1 & 100 & 1 \\
\hline Total & 8 & 17 & 12 & 37 & 55 & 68 \\
\hline
\end{tabular}

Tabla 3. Número de especies de frailejones presentes en Colombia y que se encuentran amenazados 20

\begin{tabular}{|l|l|l|}
\hline Nombre común & Especie & Familia \\
\hline Cedro & $\begin{array}{l}\text { Cederla } \\
\text { odorata }\end{array}$ & Meliaceae \\
\hline Abarco & $\begin{array}{l}\text { Cariniana } \\
\text { pyriformis }\end{array}$ & Lecythidaceae \\
\hline Roble & $\begin{array}{l}\text { Quercus } \\
\text { humbolditii }\end{array}$ & Fagaceae \\
\hline Caoba & $\begin{array}{l}\text { Swietenia } \\
\text { macrophyla }\end{array}$ & Meliaceae \\
\hline Comino & Aniba perutilis & Lauraceae \\
\hline
\end{tabular}

Comino Bistua Revista de lar Facultad de Ciencias Basicas .Universidad de Pamplona. ISSN 0120-4211

\begin{tabular}{|c|c|c|c|c|c|c|c|}
\hline Especies & Total & $\begin{array}{l}\text { Dens } \\
\text { relativa }\end{array}$ & Frec & $\begin{array}{l}\text { Frec } \\
\text { reativa }\end{array}$ & Domin & $\begin{array}{l}\text { Domin } \\
\text { Rel }\end{array}$ & IVI \\
\hline $\begin{array}{l}\text { Arcytophyllum } \\
\text { nitidum }\end{array}$ & 72 & 20,06 & 3 & 18,75 & 383,15 & 25,78 & 64,5 \\
\hline $\begin{array}{l}\text { Baccharis } \\
\text { rupícola }\end{array}$ & 80 & 22,28 & 3 & 18,75 & 63,93 & 4,30 & 45,3 \\
\hline Hypericum-sp & 62 & 17,27 & 1 & 6,25 & 255,24 & 17,18 & 40,70 \\
\hline $\begin{array}{l}\text { Hypericum } \\
\text { cardonae }\end{array}$ & 29 & 9,06 & 1 & 6,25 & 273,41 & 18,40 & 32.73 \\
\hline Espeletia-sp & 20 & 5,57 & 1 & 6,25 & 258,47 & 17,39 & 29,21 \\
\hline $\begin{array}{l}\text { Hypericum } \\
\text { gleasonii }\end{array}$ & 41 & 11,42 & 1 & 6,25 & 27,44 & 1,85 & 19,52 \\
\hline $\begin{array}{l}\text { Gaultheria } \\
\text { myrsinoides }\end{array}$ & 20 & 5,57 & 1 & 6,25 & 81,06 & 5,45 & 17,28 \\
\hline $\begin{array}{l}\text { Vaccinium } \\
\text { floribundum }\end{array}$ & 9 & 2,51 & 1 & 6,25 & 45,84 & 3,06 & 11,84 \\
\hline $\begin{array}{l}\text { Gaultheria } \\
\text { anastomosans }\end{array}$ & 7 & 1,95 & 1 & 6,25 & 47,49 & 3,20 & 11,40 \\
\hline $\begin{array}{l}\text { Hypericum } \\
\text { mexicanum }\end{array}$ & 9 & 2,51 & 1 & 6,25 & 11,46 & 0,77 & 9,53 \\
\hline Vaccinium-sp & 8 & 2,23 & 1 & 6,25 & 14,06 & 0,95 & 9,42 \\
\hline $\begin{array}{l}\text { Hypericum } \\
\text { suniperinum }\end{array}$ & 2 & 0,56 & 1 & 6,25 & 24,47 & 1,65 & 8,45 \\
\hline Nogal & \multicolumn{2}{|c|}{$\begin{array}{l}\text { Juglans } \\
\text { neotropica }\end{array}$} & \multicolumn{2}{|c|}{ Juglandaceae } & & & \\
\hline Caracoli & \multicolumn{2}{|c|}{$\begin{array}{l}\text { Anarcadium } \\
\text { excelsum }\end{array}$} & \multicolumn{2}{|c|}{ Anacardiaceae } & & & \\
\hline Carreto & \multicolumn{2}{|c|}{$\begin{array}{l}\text { Aspidosperma } \\
\text { polyneuron }\end{array}$} & \multicolumn{2}{|c|}{ Apocynaceae } & & & \\
\hline $\begin{array}{l}\text { Pino } \\
\text { colombiano }\end{array}$ & \multicolumn{2}{|c|}{$\begin{array}{l}\text { Podocarpus } \\
\text { oleifolius }\end{array}$} & \multicolumn{2}{|c|}{ Podocarpaceae } & & & \\
\hline
\end{tabular}

Tabla 4. Especies con mayor grado de amenaza, según los reportes de las CAR. ${ }^{19}$ 
